\title{
PCR-based method for targeting 16S-23S rRNA intergenic spacer regions among Vibrio species
}

\author{
Maria Hoffmann ${ }^{1,2^{*}}$, Eric W Brown ${ }^{1}$, Peter CH Feng ${ }^{1}$, Christine E Keys ${ }^{1}$, Markus Fischer ${ }^{2}$, Steven R Monday ${ }^{1}$
}

\begin{abstract}
Background: The genus Vibrio is a diverse group of Gram-negative bacteria comprised of 74 species. Furthermore, the genus has and is expected to continue expanding with the addition of several new species annually. Consequently, it is of paramount importance to have a method which is able to reliably and efficiently differentiate the numerous Vibrio species.

Results: In this study, a novel and rapid polymerase chain reaction (PCR)-based intergenic spacer (IGS)-typing system for vibrios was developed that is based on the well-known IGS regions located between the $16 \mathrm{~S}$ and $23 \mathrm{~S}$ rRNA genes on the bacterial chromosome. The system was optimized to resolve heteroduplex formation as well as to take advantage of capillary gel electrophoresis technology such that reproducible analyses could be achieved in a rapid manner. System validation was achieved through testing of 69 archetypal Vibrio strains, representing 48 Vibrio species, from which an 'IGS-type' profile database was generated. These data, presented here in several cluster analyses, demonstrated successful differentiation of the 69 type strains showing that this PCR-based fingerprinting method easily discriminates bacterial strains at the species level among Vibrio. Furthermore, testing 36 strains each of $V$. parahaemolyticus and $V$. vulnificus, important food borne pathogens, isolated from a variety of geographical locations with the IGS-typing method demonstrated distinct IGS-typing patterns indicative of subspecies divergence in both populations making this technique equally useful for intraspecies differentiation, as well.

Conclusion: This rapid, reliable and efficient IGS-typing system, especially in combination with 165 rRNA gene sequencing, has the capacity to not only discern and identify vibrios at the species level but, in some cases, at the sub-species level, as well. This procedure is particularly well-suited for preliminary species identification and, lends itself nicely to epidemiological investigations providing information more quickly than other time-honoured methods traditionally used in these types of analyses.
\end{abstract}

\section{Background}

Vibrio infections are becoming more and more common worldwide. The United States Centers for Disease Control and Prevention (CDC) estimates that 8,028 Vibrio infections and 57 deaths occur annually in the United States. Of these infections, 5,218 are foodborne in origin [1]. Three major syndromes, gastroenteritis, wound infection, and septicema, are caused by pathogenic vibrios. Within the genus Vibrio, V. cholerae, V. parahaemolyticus and $V$. vulnificus have long been established as important human pathogens in various parts of

\footnotetext{
* Correspondence: maria.hoffman@fda.hhs.gov

'Division of Microbiology, Office of Regulatory Science Center for Food Safety and Applied Nutrition, US Food and Drug Administration, Paint Branch Parkway, College Park, MD 20740, USA
}

the world. Generally, these organisms are contracted after the patient has consumed raw or undercooked seafood, such as oysters, shrimp, and fish [2]. Hence, identification and subtyping of Vibrio isolates are of significant importance to public health and the safety of the human food supply.

In the last several years, an explosion of taxonomic studies have defined and redefined the members of the genus Vibrio. In 2004, Thompson et al. [2] introduced a classification strategy for vibrios that recommended, based on concatenated 16S rRNA gene sequencing, recA, and rpoA gene sequences, that the family Vibrionaceae be separated into four new families, Vibrionaceae, Salinivibrionaceae, Photobacteriaceae and Enterovibrionaceae. The new family Vibrionaceae is 
comprised solely of the genus Vibrio, which at that time consisted of 63 distinct species. To date, the genus Vibrio has expanded to include a total of 74 distinct species http://www.vibriobiology.net/ with several new Vibrio species being identified in the last four years [3-6]. As it likely that this trend will continue, it becomes increasingly important to have simple yet accurate identification systems capable of differentiating all Vibrio species.

An array of phenotypic and genomic techniques has become available for the identification of vibrios. Biochemical characteristics have been used to identify Vibrio species. However, Vibrio and other closely related species show similar phenotypic features and, subsequently, are not easily distinguished biochemically [7]. Studies in the past have shown that identification systems based on molecular genetic techniques, such as 16S rRNA gene sequencing, 16S-23S rRNA IGS regions, amplified fragment length polymorphism (AFLP) and multilocus sequence analyses (MLSA), are more discriminating than phenotypic methods and often provide more accurate taxonomic information about a particular strain [8-11]. Several investigators have used 16S rRNA gene sequences to study overall phylogenetic relationships of the Vibrionaceae $[10,12,13]$. However, within the genus Vibrio, many different species contain nearly identical 16S rRNA gene sequences rendering this method less reliable. Furthermore, as the number of known Vibrio species continues to rise, it becomes even more likely that sequence variation in the 16S rRNA gene will no longer be sufficient alone as a target for differentiation of closely related Vibrio species or subgroups within the same species [2]. Given the apparent short-comings of $16 \mathrm{~S}$ rRNA gene sequence analyses for determining taxonomic and phylogenetic relationships of vibrios, an increasing premium is placed on the design, optimization, and deployment of subtyping schemes capable of more robust differentiation of vibrios. For bacteria with more than one rRNA operon, characterization of the 16S-23S rRNA IGS regions has been used successfully for subtyping closely related species. Due to variability in size and sequence of multiple IGS segments, size separation of PCR products spanning the IGS can enable effective differentiation of Vibrio species $[14,15]$.

Previous studies using IGS fingerprinting have encountered several problems. Foremost is the formation of heteroduplex DNA artifacts (i.e., double-stranded DNA molecules comprised of individual strands arising from two separate PCR products that share significant homology such that annealing occurs) that make interpretation of results difficult and often intangible [16-19]. Furthermore, the earlier studies often relied on either agarose or polyacrylamide gel electrophoresis (PAGE) for resolution of amplicons, making the procedure a timely process, as well [20]. In this study, we present a novel PCR-based protocol that utilizes the IGS locus along with custom-designed, Vibrio-specific $16 \mathrm{~S}$ and $23 \mathrm{~S}$ rRNA gene PCR primers for the discrimination of Vibrio species. This improved system successfully eliminated the heteroduplexes frequently encountered in other IGS-typing protocols. Moreover, the system takes advantage of capillary gel electrophoresis technology for amplicon resolution in a more rapid and accurate manner than traditional gel electrophoresis-based approaches. An analysis of 69 Vibrio type strains demonstrated that the procedure reliably differentiates at the species level, allowing the generation of an IGStype pattern database that could be used for subsequent identification of unknown Vibrio isolates. Finally, analysis of a collection of $V$. parahaemolyticus and $V$. vulnificus strains isolated from a variety of distinct geographical locales demonstrated intra-species IGS heterogeneity indicating that this protocol not only reliably differentiates at the species level but also at the subspecies level to some extent. Collectively, this report presents a Vibrio typing system that is versatile not only in identification of unknown isolates but also for epidemiological investigations, as well.

\section{Results}

The study began by confirming that the 69 Vibrio type strains obtained from American Type Culture Collection (ATCC) and the Belgian Co-Ordinated Collection of Micro Organisms (BCCM) used in this study were correctly identified. The $16 \mathrm{~S}$ rRNA gene sequence from each strain was successfully amplified and sequenced using eight additional sequencing primers. After contig assembly, BLAST (basic local alignment search tool) analysis of each product confirmed the actual identification of every type strain used in this study.

\section{Optimization and efficacy of the IGS-typing protocol}

Following identity confirmation, strains were subjected to the IGS-typing procedure designed in this study. Using the optimized PCR protocol, IGS amplicons were successfully generated from all Vibrio strains. These products were resolved using the Agilent BioAnalyzer 2100 capillary gel electrophoresis system. The system effectively separated the products, however, artifacts emerged that were not consistent with the products that should have been generated, as determined from nucleotide sequences available at the National Center for Biotechnology Information (NCBI) database. Presumably, these artifacts were a consequence of heteroduplex formation, a problem frequently associated with this type of analysis $[16,19]$. To circumvent this problem, a brief second-round amplification step was introduced that 


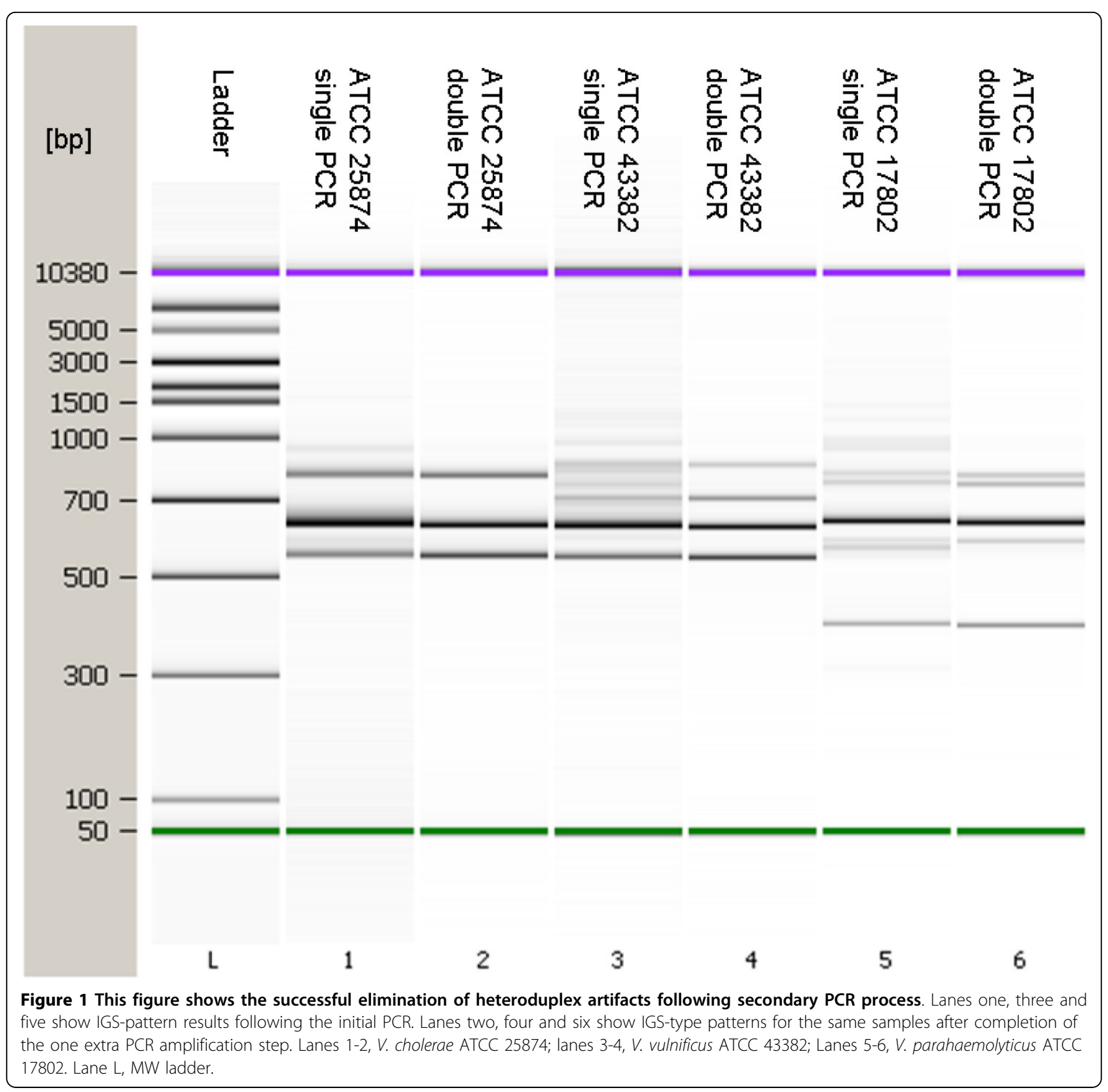

easily eliminated artifacts to produce crisp and resolute data patterns with the Agilent system (Figure 1). Analysis using BioNumerics yielded an unweight pair group method with arithmetic mean (UPGMA) dendrogram that demonstrated that the patterns generated were sufficiently different from one another so that all species could be separated by virtue of their own unique "species-specific" IGS-type patterns (Figure 2). Furthermore, these data buttress the notion that such a method focusing on the variable IGS regions of Vibrio species can be used to rapidly identify and distinguish individual species of important Vibrio pathogens.
Having demonstrated the efficiency of this method, the next step was to evaluate its fidelity. To this end, DNA was isolated from $V$. cholerae ATCC 25874, $V$. vulnificus ATCC 43382 and $V$. parahaemolyticus ATCC 17802 four separate times and individually processed (i.e., four individual biological replicates were produced). The cleaned PCR products from each of these replicates were analyzed simultaneously on the Bioanalyzer 2100 . The resulting electropherograms and gel images generated by the Bioanalyzer 2100 revealed that all DNA templates derived from the same strain reproducibly yield the same IGS-type patterns (Figure 3). Furthermore, 


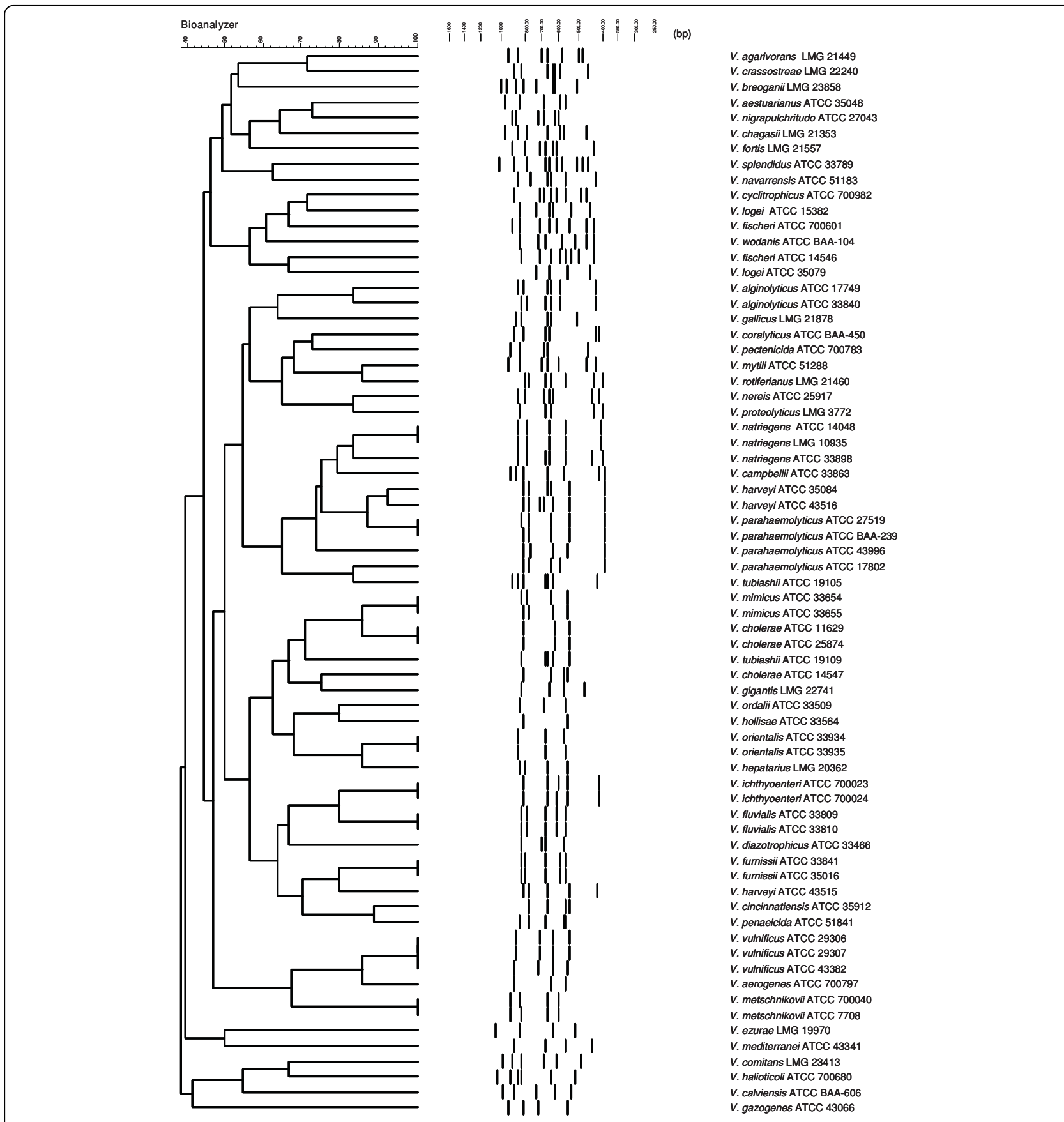

Figure 2 BioNumerics-derived UPGMA Dendrogram generated from the results of the IGS-typing procedure using 69 Vibrio reference strains. It is shown that all different species could be separated by virtue of their own unique 'specific-specific' IGS-type patterns. Parameters used to produce the dendrogram were: Dice (Opt:1.00\%) (Tol 0.25-0.25\%) (H>0.0\% S>0.0\%) [0.0\%-100.0\%].

having found that these four species consistently yielded the same IGS-type patterns, the Vibrio type strains originally tested were subjected to an additional round of testing to assure that those patterns originally observed for the type strains were also consistently reproduced. As expected, the second round of testing yielded patterns identical to those originally observed. Clearly, based on these data, the method is both efficient and reliable.

\section{Differentiation of type strains by IGS-typing analysis}

The 69 archetypal Vibrio strains used in this study represented 48 distinct species. In the course of evaluating these strains, it was noted in several cases that 


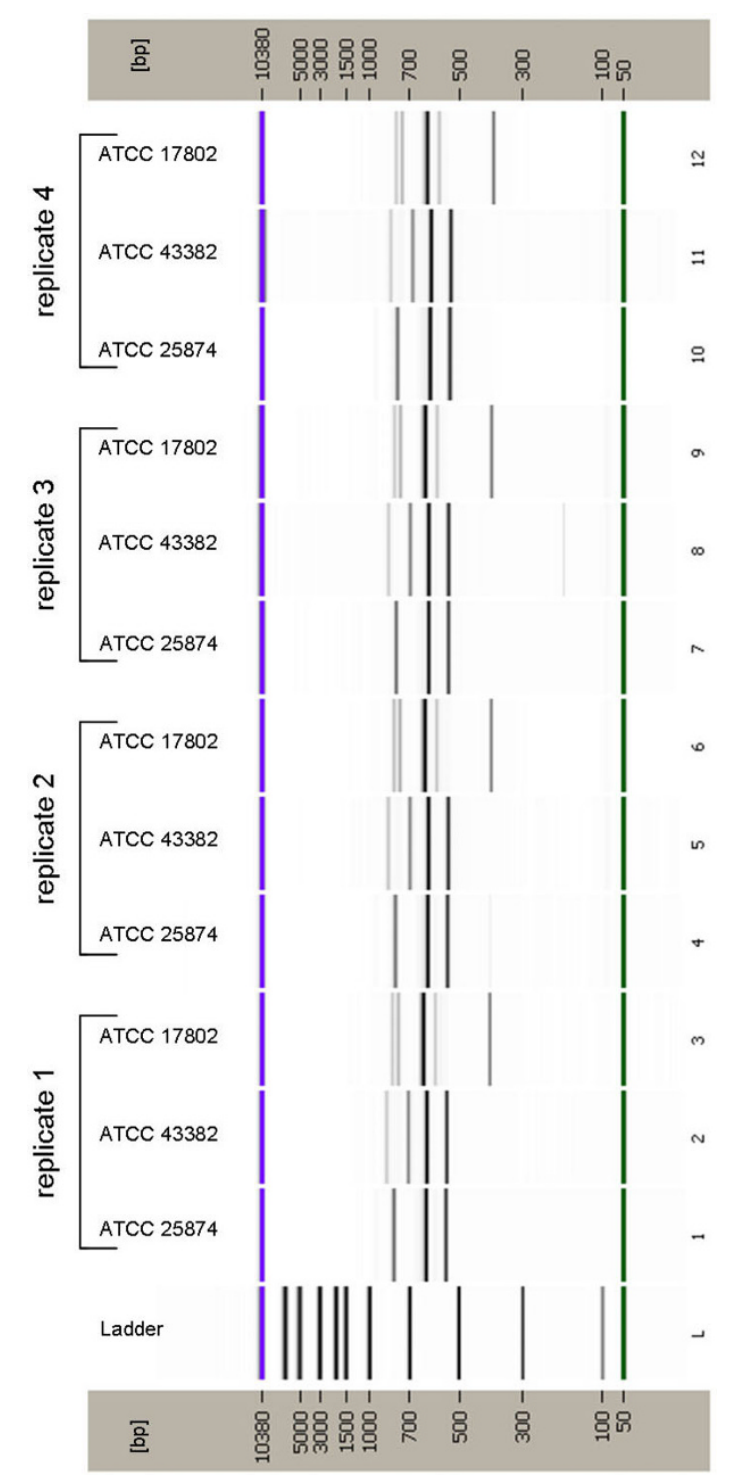

Figure 3 Virtual gel picture of IGS-type patterns obtained from replicate analyses. DNA was isolated from each strain four separate times and individually processed and evaluated for consistency in banding pattern. Lanes 1-3, replicate 1; Lanes 4-6, replicate 2; Lanes 7-9, replicate 3 and Lanes 10-12, replicate 4. Lanes 1, 4, 7 and 10: $V$. cholerae ATCC 25874; Lanes 2, 3, 8, and 11: V. vulnificus ATCC 43382; Lanes 3, 6, 9 and 12: V. parahaemolyticus ATCC 17802; Lane L, MW ladder.

distinctly different IGS-patterns were obtained from the same species having homogenous $16 \mathrm{~S}$ rRNA gene structure. For instance, $V$. natriegens ATCC 33898 differed by only a single base pair in $16 \mathrm{~S}$ rRNA gene sequence structure from $V$. natriegens strains ATCC 14048 and LMG 10935 yet produced an IGS-pattern distinctly different than that observed for either ATCC 14048 or LMG 10935, both of which yielded identical IGS fingerprints (Figure 2). Similarly, V. fischeri strains ATCC
700601 and ATCC 14546 differed by only two base pairs in 16S rRNA gene structure but also demonstrated distinctly different IGS-patterns (Figure 2). However, these latter IGS-typic differences were not entirely unexpected, as several phenotypic differences between the isolates were also noted. For instance, ATCC 14546 demonstrated a phosphorescent phenotype on Photobacterium Agar (Sigma-Aldrich Laboratories, St. Louis, MO, USA) not noted by the ATCC 700601 strain. As with the $V$. natriegens and $V$. fischeri strains, $V$. cholerae strains ATCC 14541, ATCC 11629 and ATCC 25847 also shared identical 16S rRNA gene sequence homogeneity yet produced IGS-patterns that separated the strain ATCC 14541 away from the other two strains (ATCC 11629 and ATCC 25847). This might reflect the fact that ATCC 14541 was originally deposited with ATCC as $V$. albensis and later, erroneously, reclassified as $V$. cholera as a consequence of $16 \mathrm{~S}$ rRNA gene sequence composition.

\section{Evidence of intra-species divergence by IGS-typing analysis}

To further explore the extent of this intra-species divergence phenomenon, 36 strains of $V$. parahaemolyticus and $V$. vulnificus, obtained from various geographical locations, were evaluated by this IGS-typing method. Interestingly, a significant degree of heterogeneity in the IGS-pattern obtained from the V. parahaemolyticus isolates was observed, where the UPGMA analysis separated the $V$. parahaemolyticus strains into five distinct clusters (Figure 4). These clusters were more clearly observed in a 3D multidimensional scaling (MDS) analysis (Figure 5). In this view, distinct genetic partitions were noted, separated by substantial divergence among IGS-type patterns.

Similarly, although, to a lesser extent, the $V$. vulnificus strains demonstrated IGS-pattern heterogeneity that UPGMA analysis partitioned into four distinct clusters (Figure 5 and 6). Two of these four clusters were comprised of one strain, each signaling rare and unique genotypes for these patterns. Based on the limited population examined, it is notable that the four clusters can be easily distinguished since the IGS-types are substantially diverged and largely unique both in band composition and in major size shifts. A good example is pattern cluster one, which retains a band uniquely missing in pattern four (Figure 6).

\section{Discussion}

The Vibrio genus is a complex group of marine-associated bacteria currently comprised of 74 species. The genus appears to be poised for continued growth as novel species are added regularly http://www.vibriobiology.net/. Consequently, this study was undertaken to 


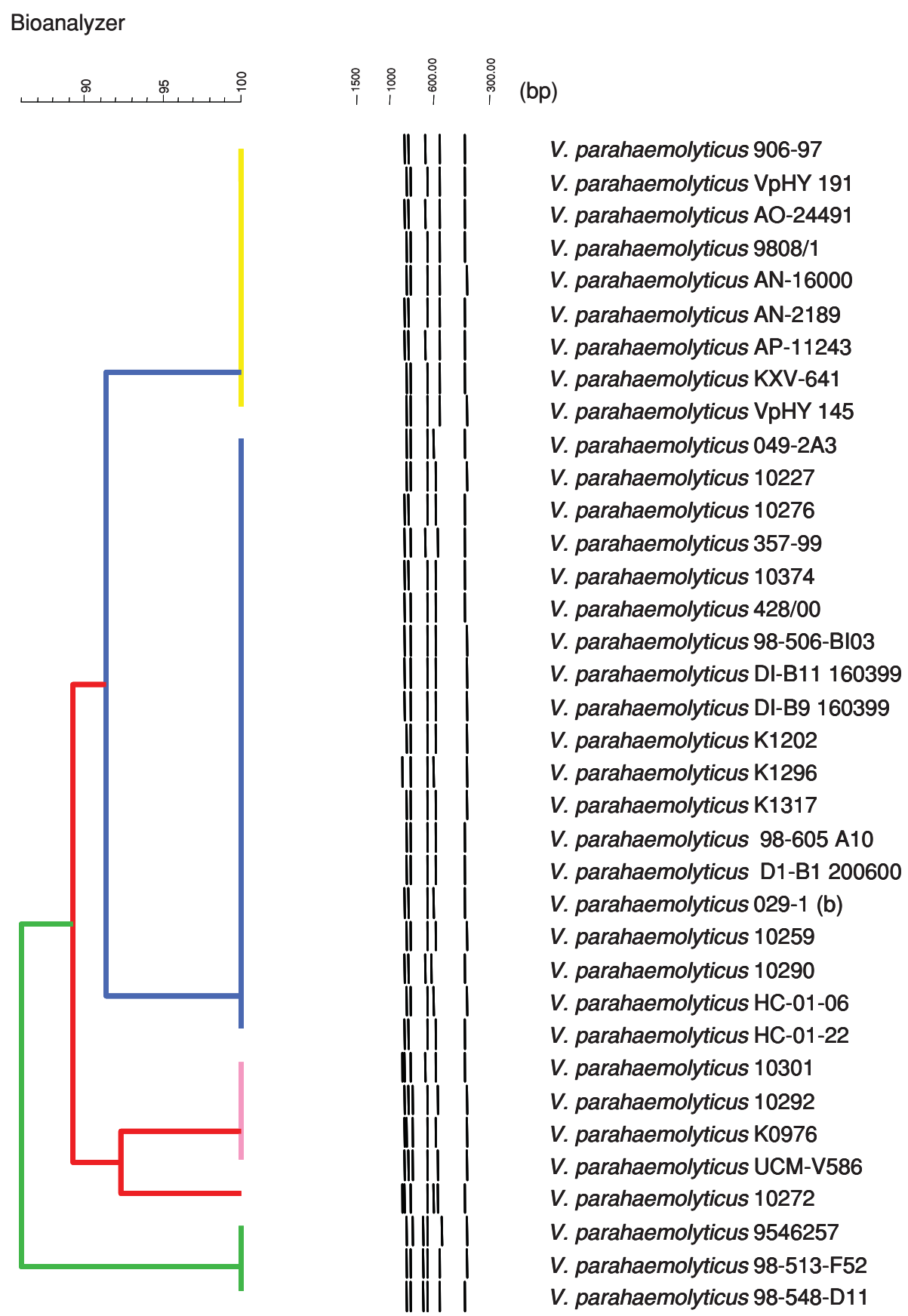

Figure 4 BioNumerics-derived UPGMA dendrogram depicting results obtained from IGS-typing of the 36 Vibrio parahaemolyticus strains. The UPGMA analysis separated the $V$. parahaemolyticus strains into five distinct clusters. Parameters used to produce the dendrogram were: Dice. (Opt:1.00\%) (Tol 0.55\%-0.55\%) (H>0.0\% S>0.0\%) [0.0\%-100.0\%].

develop a means by which these species could be efficiently, reliably, and accurately identified and differentiated. To date, analyses of IGS located between the 16S-23S rRNA gene loci have drawn considerable attention as one such means to accomplish this particular goal. Unfortunately, these analyses tend to be more laborious (i.e., restriction endonuclease analysis followed by probe-based detection) requiring a considerable time commitment. Moreover, many of these protocols generate extraneous artifacts that make interpretation of results often times difficult and unreliable.

To date, the most commonly used primers for the amplification of the IGS have been those described by Jensen et al. [21]. The 16S rRNA gene primer (G1) was 


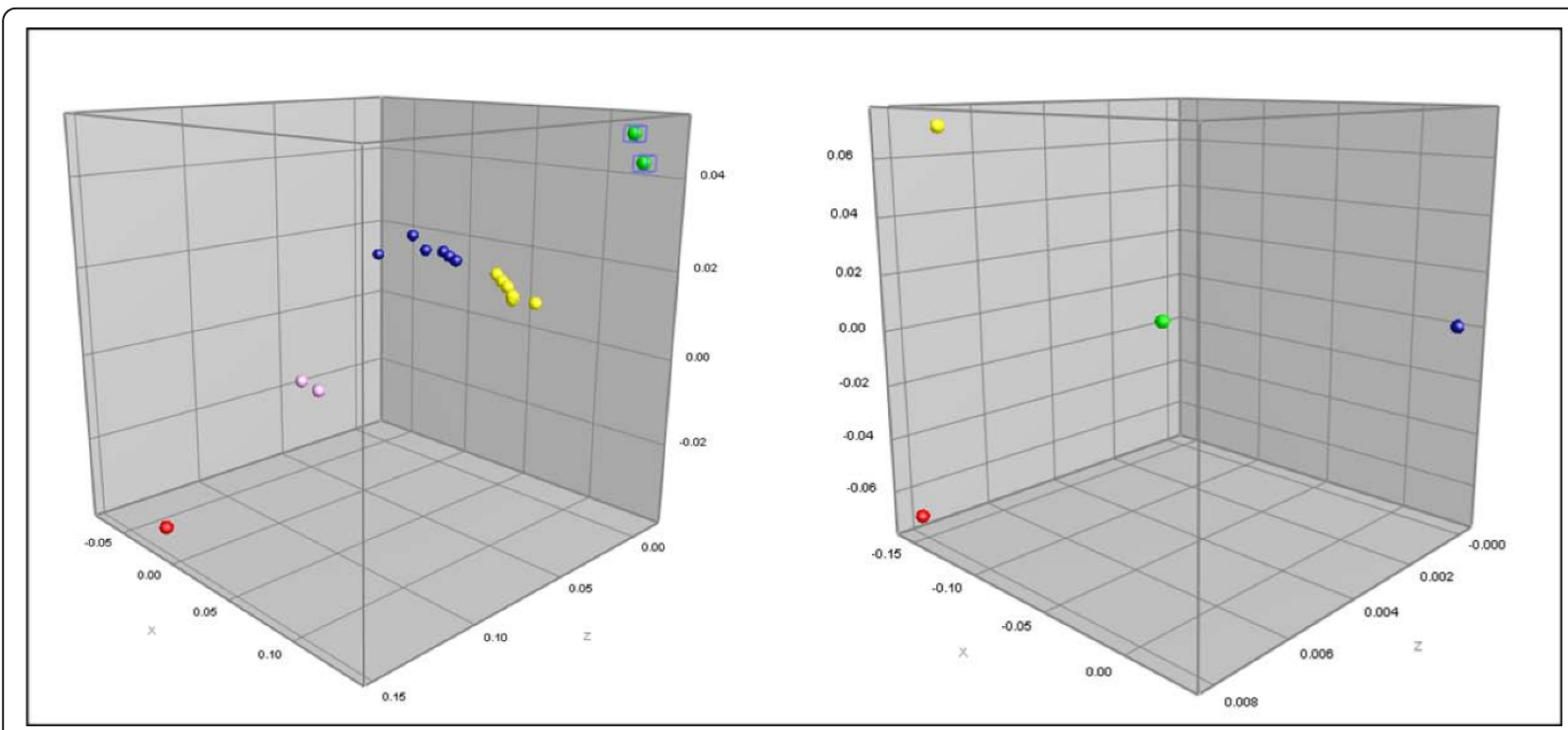

Figure 5 BioNumerics-derived MDS representing results shown in UPGMA dendrogram of $V$. parahaemolyticus and $V$. vulnificus. The graphs shown of V.parahaemolyticus (Figure 4) and V. vulnificus (Figure 6) are depicted in a 3-dimensional format to better illustrate the genetic divergence between discrete clusters. $V$. parahaemolyticus is shown in the MDS on the left, while the MDS presented on the right is for V. vulnificus.

generated for a highly conserved region of the $16 \mathrm{~S}$ rRNA gene locus approximately 30-40 bp upstream of the IGS using the 16S rRNA gene sequence data generated by Dams et al [22] from a broad range of bacterial and eukaryotic genera (107 species). In contrast, as the $23 \mathrm{~S}$ rRNA gene sequence is much less conserved than that of the $16 \mathrm{~S}$ rRNA gene, the $23 \mathrm{~S}$ primer (L1) was designed from the 23S rRNA gene sequences of only five bacterial and four plant species previously determined by Gutell et al [23]. As these primers were not based solely on Vibrio $16 \mathrm{~S}$ and $23 \mathrm{~S}$ rRNA gene sequences, a new set of Vibrio-specific primers was designed from an alignment of $16 \mathrm{~S}$ and $23 \mathrm{~S}$ Vibrio rRNA gene sequences. PCR reactions were optimized using these primers such that the amplification products from four reference strains ( $V$. parahaemolyticus BAA239 (O3:K6), V. cholerae ATCC 25874, V. vulnificus ATCC 43382 and $V$. fischeri ATCC 700601) were consistent with the number and sizes of those that could be theoretically derived from genomic sequences available at the NCBI database (V. parahaemolyticus RIMD 2210633 (Chromosome I: NC_004603; chromosome II: NC_004605), $V$. cholerae O395 (chromosome 1: NC_009456; chromosome 2: NC_009457), V. vulnificus CMCP6 (chromosome 1: NC_004459; chromosome 2: NC_004460) and V. fischeri ES 114 (chromosome 1: NC_006840; chromosome 2: NC_006841)). As an example, the chromosome coordinates, relative size, and number of IGS regions targeted by this assay for $V$. parahaemolyticus, $V$. vulnificus, and $V$. cholerae are depicted in Figure 7. In every case, IGS banding patterns correlated perfectly with expected fragment size (compare Figure 7 to Figures 1 and 3). Afterwards, the testing of each remaining reference species demonstrated unique banding patterns for all strains included. In the course of the optimization procedure, artifacts, the likely result of heteroduplex formation, were universally noted in the reaction products derived from these species. While several means by which heteroduplex formation could be eliminated or reduced are discussed in numerous publications $[16,18,19]$, we found that only one [24], with some modification, produced results acceptable for use in this particular protocol. Subsequently, PCR products derived from the first amplification procedure were processed further with a second round of PCR optimized for heteroduplex elimination. Numerous testing of the two-round PCR procedure repeatedly yielded products devoid of transient artifacts, confirming that the process was suitable for and highly compatible with this type of analysis.

Previous IGS studies have relied on either agarose or PAGE for resolution of the amplicons generated by PCR-based IGS-typic analyses [14,25]. These methods can be somewhat cumbersome and require a lengthy amount of time to perform. To overcome this limitation, this protocol was engineered to take advantage of the rapid and sensitive capillary gel electrophoresis technology. Using the Agilent BioAnalyzer 2100 system, it was determined that a minimal amount of effort to more thoroughly clean the second round PCR products 
allowed this technology to deliver results that were at least as good as, if not better than, those obtained from traditional electrophoresis protocols. Furthermore, the Agilent system provided the additional benefit of a highly accurate and easily interpreted virtual gel-based result. That is, band interpretations were based on real genotypic differences defined by obvious deviations in band size, rather than subjective band 'bin' assignments so often incorporated with conventional agarose and PAGE.
While all reference species tested produced results that sufficiently differentiated them, as noted by the cluster analyses, we also determined, in a few cases, identical species having homogenous $16 \mathrm{~S}$ rRNA gene sequence structure produced different IGS-type banding patterns. These patterns were often times substantially different such that identical species were separated widely on the resultant dendrograms. One explanation for this observation is that horizontal gene transfer (HGT) has occurred in the IGS of one isolate causing a 


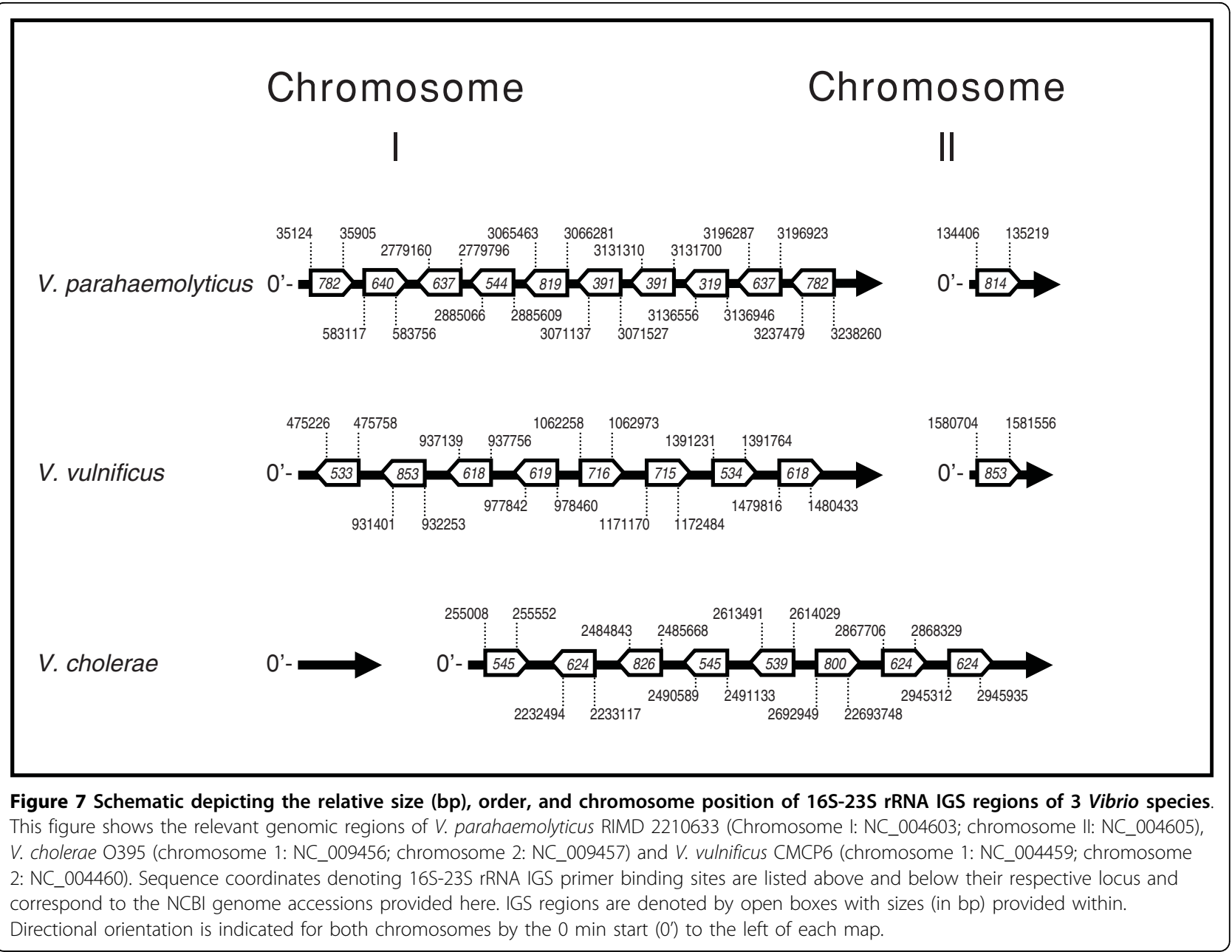

deviation in its specific IGS-typic pattern. Additionally, as might be the case for the $V$. fischeri isolates that have identical 16S rRNA gene structure and, presumably, are the same species, when, in fact, they are not. In this particular case, one of the isolates displayed a phosphorescent phenotype while the other did not. Similarly, $V$. cholerae isolates demonstrated that all had identical 16S rRNA gene sequence structures yet only two of the isolates produced identical IGS-prints. The third V. cholerae ATCC 14541 produced a distinctly different banding pattern. Of particular interest is that this 'atypical' $V$. cholerae strain was originally deposited as $V$. albensis, not $V$. cholerae, underscoring the risks associated with speciating strains based solely on their $16 \mathrm{~S}$ rRNA gene structure.

To explore intraspecies level IGS-typic divergence for several important Vibrio species, a comprehensive study characterizing the IGS-typic relationship within a population of $36 \mathrm{~V}$. parahaemolyticus and $36 \mathrm{~V}$. vulnificus isolates derived from different geographic locations was performed. As expected, these strains confirmed divergence of IGS-type patterns at the intraspecies level. Surprisingly, 15 different $V$. parahaemolyticus IGS-types, consisting of up to seven bands each, partitioned readily into five distinct clusters. This particular observation deviated significantly from an earlier study [26] proposing that $V$. parahaemolyticus was segregated into four clusters based solely on the four distinct IGS-patterns that were observed in their PAGE analysis. This significant difference in segregative ability allows a more powerful and discriminatory resolution of strains at the intraspecies level. Furthermore, the previous study [26], suggests that mismatches in the L1 (Jensen) primer [21] gave rise to a different and, presumably, an incorrect banding pattern from that generated when using their own primer set, although the L1/G1 pattern they present in their representation is clearly in agreement with the pattern that would be theoretically obtained from the NCBI genomic sequence of the $V$. parahaemolyticus RIMD 2210683 strain. Interestingly, the L1/G1 pattern presented in the earlier Jensen et al. study is entirely consistent with that of our own work, which is not 
entirely surprising as the sequence of L1 (and G1) are $100 \%$ complementary to the annealing sites of all 11 V. parahaemolyticus RIMD 2210683 rDNA loci.

We found in preliminary investigations of $V$. vulnificus that, although not to the degree of $V$. parahaemolyticus, the IGS-typing data also consisted of numerous $(\sim 10)$ unique patterns that partitioned nicely into four distinct clusters. Moreover, several of these isolates produced IGS-prints that consisted of five to six bands, significantly deviating from the pattern produced by our reference strains ( $V$. vulnificus ATCC 43382, ATCC 29307 and ATCC 29306), as well as the theoretical pattern derived from the NCBI genomic sequence submission for $V$. vulnificus CMCP6 (NC_004459 and NC_004460), all of which consisted of a four band IGS-type pattern. These data may signal a reticulate evolutionary pattern for IGS sequences in this group of vibrios.

Notably, we found that the IGS-typing data derived from the $V$. parahaemolyticus study correlated nicely with the distributions of MLST sequence types (STs) previously generated for these strains, with no single ST observed in more than one cluster [27]. This finding was also noted in the $V$. vulnificus analysis [28]. For example, strains having ST16 converged into ribotype cluster one. Additionally in the case of $V$. parahaemolyticus, it is interesting to note that clusters two, three, four and five were primarily comprised of United Statesderived isolates, indicating some degree of phylogeographic concordance with resultant IGS-prints (Figure 4). Taken together these observations suggest that it may, indeed, be possible to engage in epidemiological studies of outbreak strains using IGS-typing methodology. Furthermore, understanding and characterizing the relationship of these outbreak strains to their environmental counterparts might also be facilitated using this analytical strategy.

At present, it appears that, in complex genera consisting of numerous species, identification by monotypic analysis becomes increasingly more difficult and unreliable [2]. Clearly, this is the case for 16S rRNA gene sequence analysis of Vibrio strains, where unique and distinct species retain virtually identical $16 \mathrm{~S}$ rRNA gene sequences, differing by as little as two to three ( $\geq 0.2 \%)$ base pairs. However, we have shown that it may be possible to discriminate at the species and intra-species levels using an analysis of IGS regions that is easy to perform, avoids cumbersome and time-consuming PAGE and agarose gel electrophoresis technologies and is devoid of the interfering artifacts that make accurate interpretation of results difficult at best. Moreover, this strategy incorporates a conservative analytical approach where only substantial, non-ambiguous results are considered in the interpretation of the analysis. In combination with a $16 \mathrm{~S}$ rRNA gene sequencing analysis, the approach becomes even more powerful in the identification of species and, consequently, should prove invaluable for differentiation of species within a very complex Vibrio genus and for characterization of outbreak strains and isolates found in suspect environmental/food samples.

\section{Conclusion}

This report describes a method that discriminates Vibrio species in a rapid and accurate manner. PCR amplification products derived from the 16S-23S rRNA genes IGS region could be analyzed using capillary gel electrophoresis technology to generate an IGS-typing pattern for each strain tested. The study showed that each of the species produced an IGS-typing pattern unique to itself that could be used to identify Vibrio species. Furthermore, the analysis could be performed more rapidly with the capillary gel electrophoresis procedure than either traditional agarose or PAGE protocols. The study also shows that there is sufficient intra-species IGS-typing pattern variation that differentiates at the subspecies, as well, especially when used in combination with $16 \mathrm{~S}$ rRNA gene sequencing. As such, the procedure described in this report could be successfully used in preliminary epidemiological investigations, as well as other studies, to yield information more rapidly than other established subtyping methods requiring a considerably greater time commitment, such as pulsed field gel electrophoresis (PFGE), AFLP or MLSA.

\section{Methods}

\section{Bacterial Strains, Growth Condition and Characterization}

The 69 Vibrio type strains listed in Table 1 represented 48 species that served as reference taxa for this study. Isolates were obtained from ATCC and BCCM. Freezedried (lyophilized) cultures were revived according to protocols provided by the ATCC and BCCM curators. 16S rRNA gene sequencing (Amplicon Express, Pullman, WA, USA) was used as confirmation in assuring the identity of reference strains.

Thirty six $V$. parahaemolyticus and $36 \mathrm{~V}$. vulnificus strains from various laboratories within the Food and Drug Administration (FDA) were also selected for this study. These strains, listed in Table 2, were very well characterized at the FDA (Dauphin Island AL) $[20,27]$. The strains were grown overnight with shaking (112 $\mathrm{rpm}$ ) in Luria Bertani (LB; DIFCO Laboratories) medium at $37^{\circ} \mathrm{C}$. Thiosulfate-Citrate-Bile Salts-Sucrose (TCBS; DIFCO Laboratories) Agar was used also as a selective agar to differentiate $V$. vulnificus and $V$. parahaemolyticus strains. Further confirmation of strain identity based on biochemical identification was performed using the standardized API 20 E identification system (bioMérieux, L'Etoile, France) and the Pathotec ${ }^{\mathrm{R}}$ 
Table 1 ATCC and BCCM type strain collection used in this study

\begin{tabular}{|c|c|c|c|}
\hline Designation & Strain* & Designation & Strain* \\
\hline ATCC 700797 & $V$. aerogenes & ATCC 33898 & V. natriegens \\
\hline ATCC 35048 & V. aestuarianus & ATCC 14048 & V. natriegens \\
\hline ATCC 33840 & V. alginolyticus & ATCC 51183 & V. navarrensis \\
\hline ATCC 17749 & V. alginolyticus & ATCC 25917 & $V$. nereis \\
\hline ATCC BAA-606 & V. calviensis & ATCC 27043 & V. nigrapulchritudo \\
\hline ATCC 33863 & V. campbellii & ATCC 33509 & V. ordalii \\
\hline ATCC 11629 & V. cholerae & ATCC 33934 & V. orientalis \\
\hline ATCC 25874 & V. cholerae & ATCC 33935 & V. orientalis \\
\hline ATCC 14547 & V. cholerae & ATCC 43996 & V. parahaemolyticus \\
\hline ATCC 35912 & V. cincinnatiensis & ATCC 27519 & V. parahaemolyticus \\
\hline ATCC 700982 & V. cyclitrophicus & ATCC 17802 & V. parahaemolyticus \\
\hline ATCC BAA-450 & V. coralyticus & ATCC BAA-239 & V. parahaemolyticus \\
\hline ATCC 33466 & V. diazotrophicus & ATCC 700783 & V. pectenicida \\
\hline ATCC 700601 & V. fischeri & ATCC 51841 & V. penaeicida \\
\hline ATCC 14546 & V. fischeri & ATCC 33789 & V. splendidus \\
\hline ATCC 33809 & V. fluvialis & ATCC 19105 & V. tubiashii \\
\hline ATCC 33810 & V. fluvialis & ATCC 19109 & V. tubiashii \\
\hline ATCC 35016 & V. furnissii & ATCC 43382 & V. vulnificus \\
\hline ATCC 33841 & V. furnissii & ATCC 29306 & V. vulnificus \\
\hline ATCC 43066 & V. gazogenes & ATCC 29307 & V. vulnificus \\
\hline ATCC 700680 & V. halioticoli & ATCC BAA-104 & V. wodansis \\
\hline ATCC 35084 & V. harveyi & LMG 21449 & V. agarivorans \\
\hline ATCC 43515 & V. harveyi & LMG 23858 & V. breoganii \\
\hline ATCC 43516 & V. harveyi & LMG 21353 & V. chagasii \\
\hline ATCC 33564 & V. hollisae & LMG 23413 & V. comitans \\
\hline ATCC 700023 & V. ichthyoenteri & LMG 22240 & V. crassostreae \\
\hline ATCC 700024 & V. ichthyoenteri & LMG 19970 & V. ezurae \\
\hline ATCC 15382 & V. logei & LMG 21557 & V. fortis \\
\hline ATCC 35079 & V. logei & LMG 21878 & V. gallicus \\
\hline ATCC 43341 & V. mediterranei & LMG 22741 & V. gigantis \\
\hline ATCC 700040 & V. metschnikovii & LMG 20362 & V. hepatarius \\
\hline ATCC 7708 & V. metschnikovii & LMG 10935 & V. natriegens \\
\hline ATCC 33654 & V. mimicus & LMG 3772 & V. proteolyticus \\
\hline ATCC 33655 & V. mimicus & LMG 21460 & V. rotiferianus \\
\hline ATCC 51288 & V. mytili & & \\
\hline
\end{tabular}

*The following Vibrio type strains, used as reference strains in this study, were recently reclassified as different genera, as described here: $V$. calviensis became Enterovibrio calviensis [29]; V. fisheri became Aliivibrio fisheri, V. logei became Aliivibrio logei, V. wodanis became Aliivibrio wodanis [30]; and V. hollisae became Grimontia hollisae [31]. Through this paper, the former genus and species designations are used.

Cytochrome Oxidase Test (Remel, Lenexa, KS, USA) using pure cultures of isolated colonies grown on LB for $16-20$ hours at $37^{\circ} \mathrm{C}$ according to the protocol provided by suppliers. API $20 \mathrm{E}$ identification was performed using the Apiweb ${ }^{\text {tw }}$ identification software.

Genomic DNA was isolated from all strains using the ZR Fungal/Bacterial DNA kit (Zymo Research, Orange, CA) according to the manufacturer's protocol. Purified DNA was quantified spectrophotometrically using a Nano Drop-1000 Spectrophotometer (NanoDrop
Technologies, Inc., Wilmington, DE, USA) and diluted to a final concentration of $100 \mathrm{ng} / \mu \mathrm{l}$ using DNase/ RNase-free double-distilled water $\left(\mathrm{ddH}_{2} \mathrm{O}\right)$.

\section{$16 \mathrm{~S}$ rRNA gene sequencing}

Oligonucleotide primers for amplification of the $16 \mathrm{~S}$ rRNA gene and subsequent sequencing were designed using conserved sequences detected within a Clustal X nucleotide alignment of the Vibrio $16 \mathrm{~S}$ nucleotide sequences obtained from the NCBI database. 16S rRNA gene sequences from 15 separate Vibrio species were used for the sequence alignment. Derived primer sequences were evaluated for predicted efficiency using the NetPrimer computer software (Premier Biosoft International, Palo Alto, CA, USA). The primers used for PCR amplification were: 16SF [5'-GTTTGATCATGGCTCAGATTG-3'] and 16SR [5'-CTACCTTGTTACGACTTCACC-3'].

The PCR was performed in a $50 \mu \mathrm{l}$ volume with HotStarTaq Master Mix (Qiagen, Valencia, CA, USA) containing $400 \mu \mathrm{M}$ dNTP (each of dATP, dCTP, dGTP and dTTP), $5 \mathrm{U}$ of HotStart Taq Polymerase (Qiagen), 1x Taq polymerase buffer (Qiagen), $2.5 \mathrm{mM} \mathrm{MgCl}_{2}$ and a $300 \mathrm{nM}$ concentration of each primer with $\sim 100 \mathrm{ng}$ of DNA template. The optimized amplification program began with a $95^{\circ} \mathrm{C}$ for 15 min enzyme activation step. To minimize PCR products derived from mispriming events, the actual amplification was initiated with a 'touchdown' PCR step consisting of 10 cycles at $95^{\circ} \mathrm{C}$ for 30 second (sec), $72^{\circ} \mathrm{C}-63^{\circ} \mathrm{C}$ (decreasing $1^{\circ} \mathrm{C} /$ cycle) for 20 sec and $72^{\circ} \mathrm{C}$ for $1.00 \mathrm{~min}$ followed by 35 cycles of $95^{\circ} \mathrm{C}$ for $30 \mathrm{sec}, 63^{\circ} \mathrm{C}$ for $20 \mathrm{sec}$ and $72^{\circ} \mathrm{C}$ for $1.00 \mathrm{~min}$. The process was finished with a single cycle at $72^{\circ} \mathrm{C}$ for 2 min and stored at $4{ }^{\circ} \mathrm{C}$ until analyzed.

Both strands of amplified PCR products were sequenced by Amplicon Express (Pullman, WA, USA) using Big Dye chemistry with 4 forward and 4 reverse target-specific sequencing primers (Table 3 ) in an ABI 3730 XL DNA sequencer according to the manufacturer's directions. DNA sequences were edited and assembled using DNAStar, Inc. (Madison, WI) Lasergene SeqMan II 5.07 sequence analyses software. After analyzing and assembling the respective sequences, a consensus sequence was used to query the NCBI BLAST database at NCBI to reconfirm reference strain identity.

\section{IGS-typing PCR}

IGS PCR primers were designed using conserved sequences detected within a Clustal $\mathrm{X}$ nucleotide alignment of both the Vibrio 16S rRNA gene and 23S rRNA gene nucleotide sequences obtained from NCBI. The 16S rRNA gene and 23S rRNA gene sequences from 15 separate Vibrio species (i.e., V. navarrensis, V. vulnificus, 
Table 2 V. parahaemolyticus and $V$. vulnificus strains used in this study

\begin{tabular}{|c|c|c|c|c|c|c|c|}
\hline \multicolumn{4}{|c|}{ V. parahaemolyticus strains } & \multicolumn{4}{|c|}{ V. vulnificus strains } \\
\hline Strain & Country* & Source & $\mathrm{ST}^{\#}$ & Strain & Country* & Source & $\mathrm{ST}^{\#}$ \\
\hline AN-16000 & Bangladesh & Clinical & 3 & 98-783 DP-A1 & USA-LA & Environ. & 26 \\
\hline AN-2189 & Bangladesh & Clinical & 3 & 99-742 DP-A9 & USA-MS & Environ. & 22 \\
\hline $\mathrm{AO}-24491$ & Bangladesh & Clinical & 3 & 99-736 DP-C7 & USA-FL & Environ. & 34 \\
\hline AP-11243 & Bangladesh & Clinical & 51 & 99-624 DP-C10 & USA-TX & Environ. & 17 \\
\hline $428 / 00$ & Spain & Clinical & 17 & 99-779 DP-D2 & USA-LA & Environ. & 51 \\
\hline UCM-V586 & Spain & Environ. & 45 & 99-796 DP-E7 & USA-FL & Environ. & 22 \\
\hline $9808 / 1$ & Spain & Clinical & 3 & 98-640 DP-E9 & USA-LA & Environ. & 24 \\
\hline $906-97$ & Peru & Clinical & 3 & ATL 6-1306 & USA-FL & Clinical & 16 \\
\hline $357-99$ & Peru & Clinical & 19 & ATL 71503 & USA-FL & Clinical & 16 \\
\hline VpHY191 & Thailand & Clinical & 3 & ATL 9579 & USA-TX & Clinical & 19 \\
\hline VpHY145 & Thailand & Clinical & 3 & ATL 61438 & USA-TX & Clinical & N/A \\
\hline KXV-641 & Japan & Clinical & 3 & ATL 9823 & USA-LA & Clinical & 37 \\
\hline 98-605-A10 & USA-CT & Environ. & 31 & ATL 71491 & USA-TX & Clinical & 32 \\
\hline 9546257 & USA-CA & Clinical & 32 & ATL 71504 & USA-LA & Clinical & 32 \\
\hline $049-2 \mathrm{~A} 3$ & USA-OR & Environ. & 57 & BUF 7211 & USA-FL & Clinical & N/A \\
\hline 98-506-B103 & USA-VA & Environ. & 30 & DAL 8-9131 & USA-TX & Clinical & N/A \\
\hline 98-548-D11 & USA-MA & Environ. & 34 & DAL 6-5000 & USA-LA & Clinical & 18 \\
\hline $98-513-F 52$ & USA-LA & Environ. & 34 & FLA 8869 & USATX & Clinical & 40 \\
\hline DI-B9 160399 & USA-AL & Environ. & 25 & FLA 9509 & USA-LA & Clinical & 40 \\
\hline DI-B11 160399 & USA-AL & Environ. & 54 & LOS 6966 & USA-TX & Clinical & 2 \\
\hline DI-B-1 200600 & USA-AL & Environ. & 23 & LOS 7343 & USA-LA & Clinical & 32 \\
\hline HC-01-22 & USA-WA & Environ. & 43 & NSV 5736 & USA-AL & Clinical & 33 \\
\hline HC-01-06 & USA-WA & Environ. & 41 & NSV 5830 & USA-FL & Clinical & 52 \\
\hline K0976 & USA-AK & Environ. & 4 & NSV 5829 & USA-FL & Clinical & 16 \\
\hline K1202 & USA-AK & Environ. & 43 & ORL 1506 & USA-FL & Clinical & 16 \\
\hline K1296 & USA-AK & Environ. & 9 & ORL 8324 & USA-FL & Clinical & 37 \\
\hline K1317 & USA-AK & Environ. & 5 & ORL 8073 & USA-FL & Clinical & N/A \\
\hline 029-1 (b) & USA-OR & Environ. & 36 & 10152 & USA-WA & Clinical & $\mathrm{N} / \mathrm{A}$ \\
\hline 10290 & USA-WA & Clinical & 37 & 10156 & USA-WA & Clinical & N/A \\
\hline 10292 & USA-WA & Clinical & 50 & 10157 & USA-WA & Environ. & N/A \\
\hline 10227 & USA-WA & Environ. & N/A & 10158 & USA-WA & Environ. & N/A \\
\hline 10259 & USA-WA & Clinical & N/A & 10159 & USA-WA & Clinical & N/A \\
\hline 10272 & USA-WA & Environ. & N/A & 10163 & USA-WA & Environ. & N/A \\
\hline 10276 & USA-WA & Environ. & N/A & 10164 & USA-WA & Clinical & N/A \\
\hline 10301 & USA-WA & Environ. & $\mathrm{N} / \mathrm{A}$ & 10165 & USA-WA & Clinical & N/A \\
\hline 10374 & USA-WA & Clinical & $\mathrm{N} / \mathrm{A}$ & 10167 & USA-WA & Clinical & N/A \\
\hline
\end{tabular}

*USA:United States; AL: Alabama; AK: Alaska; CA: California; CT: Connecticut; FL: Florida; LA: Louisanna; MA: Maryland; MS: Mississippi; OR: Organ; TX: Texas; VA: Virginia; WA: Washington "ST: sequence type

Table 3 16S rRNA gene sequencing primers used in this study

\begin{tabular}{ll}
\hline $16 S R 1$ & $5^{\prime}$-CAATATTCCCYACTGCTGC-3' \\
$16 S R 2$ & 5'-CATCGTTTACGYCGTGGACT-3' \\
$16 S R 3$ & 5'-GCTCGTTGCGGGACTTA-3' \\
$16 S R 4$ & 5'-GCTACCTTGTACGACTTCACC-3' \\
$16 S F 1$ & 5'-GCRGGCCTAAYACATGCA-3' \\
$16 S F 2$ & 5'-TGAGACACGGYCCAGACTCCTAC-3' \\
$16 S F 3$ & 5'-GTAGCGGTGAAATGCGTAGA-3' \\
$16 S F 4$ & 5'-TGTCGTCAGCTCGTGTYGTG-3' \\
\hline
\end{tabular}

$V$. fischeri, V. logei, V. mediterranei, V. pelagius, $V$. splendidus, $V$. lentus, $V$. harveyii, $V$. parahaemolyticus, $V$. natriegens, $V$. ordalii, $V$. hollisae, $V$. fluvialis and $V$. cholerae) and $E$. coli were used for the sequence alignment. Derived primer sequences 16S.6 [5'-ACTGGGGTGAAGTCGTAACA-3'] and 23S.1 [5'-CTTCATCGCCTCTGACTGC-3'] were evaluated for predicted efficiency using the NetPrimer Computer software.

PCR was performed in a $50 \mu \mathrm{l}$ volume containing 300 $\mu \mathrm{M}$ dNTP, $5 \mathrm{U}$ of HotStart Taq Polymerase, $1 \times$ Taq polymerase buffer, $1.5 \mathrm{mM} \mathrm{MgCl} 2$ and a $300 \mathrm{nM}$ 
concentration of each primer with $100 \mathrm{ng}$ of DNA template. The amplification program was $95^{\circ} \mathrm{C}$ for 15 min, 10 cycles at $95^{\circ} \mathrm{C}$ for 30 sec., $73^{\circ} \mathrm{C}-64^{\circ} \mathrm{C}$ (decreasing $1^{\circ} \mathrm{C} /$ cycle) for $10 \mathrm{sec}$ and $72^{\circ} \mathrm{C}$ for $45 \mathrm{sec}$. Afterwards, complete amplification was achieved with 34 cycles of $95^{\circ} \mathrm{C}$ for $30 \mathrm{sec}, 64^{\circ} \mathrm{C}$ for $10 \mathrm{sec}$ and $72^{\circ} \mathrm{C}$ for $45 \mathrm{sec}$. The process was finished with a single cycle at $72^{\circ} \mathrm{C}$ for $1 \mathrm{~min}$ and stored at $4^{\circ} \mathrm{C}$.

Heteroduplex formation was resolved with an additional amplification cycle [24] where the initial PCR products were diluted 1:5 in a $30 \mu \mathrm{l}$ volume and subjected to a single amplification cycle of $95^{\circ} \mathrm{C}$ for $15.00 \mathrm{~min}, 64^{\circ} \mathrm{C}$ for $1.00 \mathrm{~min}$ and $72^{\circ} \mathrm{C}$ for $10.00 \mathrm{~min}$ in a similar reaction mixture containing $600 \mathrm{nM}$ primer concentration. Afterwards, the PCR products were purified using QIAquick PCR Purification Kit (Qiagen) according to the manufacturer's protocol and eluted into $10 \mu \mathrm{L}$ of nuclease-free Water.

\section{Analysis of IGS-typing fingerprints}

IGS PCR amplicons were resolved by capillary gel electrophoresis using the Agilent BioAnalyzer 2100 and the Agilent DNA 7500 Assay Protocol (Agilent Technologies, Inc., Santa Clara, CA, USA). Using the BioAnalyzer 2100 integrated computer software, electropherograms and gel patterns were generated depicting the resulting PCR products derived from the IGS-typing reaction. Faint bands comprising less than $5 \%$ of the total DNA concentration and measuring less than $1 \mathrm{ng} / \mathrm{ul}$ were discarded prior to performing the analysis using BioNumerics fingerprinting software 5.10 (Applied Mathematics, Sint Martens Latem, Belgium). BioAnalyzer data was imported directly into the BioNumerics software using a custom script written by Applied Math for the BioNumerics software. The BioNumerics software used the Dice similarity coefficient to generate the UPGMA dendrograms presented in this study with Dice parameters: Optimization (Opt): $1.00 \%$, Tolerance (Tol). $0.25 \%-0.25 \%$ for the reference strains, and Opt: $1.00 \%$, Tol. $0.55 \%-0.55 \%$ for the $36 \mathrm{~V}$. vulnificus and $36 \mathrm{~V}$. parahaemolyticus strains.

\section{List of abbreviations used}

AFLP: amplified fragment length polymorphism; ATCC: American Type Culture Collection; BCCM: Belgian CoOrdinated Collection of Micro-Organisms; BLAST: basic local alignment search tool; CDC: The United States Centers for Disease Control and Prevention; DNA: deoxyribonucleic acid; FDA: Food and Drug Administration; HGT: horizontal gene transfer; IGS: intergenic spacer; LB: Luria Bertani; LMG: Laboratory of Microbiology Gent Bacteria Collection; MDS: 3D multidimensional scaling; MLSA: multilocus sequence analyses; NCBI: National Center for Biotechnology Information;
Opt: Optimization; PAGE: polyacylamide gel electrophoresis; PCR: polymerase chain reaction; recA: recombinase A; rpoA: DNA-directed RNA polymerase subunit alpha; rRNA: ribosomal ribonucleic acid; ST: sequence type; S: Svedberg; TCBS: Thiosulfate-Citrate-Bile SaltsSucrose; Tol: tolerance; UPGMA: unweight pair group method with arithmetic mean; V:: Vibrio.

\section{Acknowledgements}

This project was supported by an appointment of $\mathrm{MH}$ to the Research Fellowship Program for the Center for Food Safety and Applied Nutrition administered by the Oak Ridge Associated Universities. The authors wish to thank Dr. González-Escalona for sharing his V. vulnificus and $V$. parahaemolyticus strains and for his insights in this study.

\section{Author details}

'Division of Microbiology, Office of Regulatory Science Center for Food Safety and Applied Nutrition, US Food and Drug Administration, Paint Branch Parkway, College Park, MD 20740, USA. Institute of Food Chemistry, University of Hamburg, Grindelallee 117, D-20146 Hamburg, Germany.

\section{Authors' contributions}

All authors played an integral part of project conception and method development described in the article. Each author has read and approved the final version of the manuscript. Specifically, MH performed the

experimental procedures of the method development, including subsequent validation, and optimization, as well as the data analysis and interpretation of the results, and preparation of the manuscript. PCHF assisted with the microbiology component of the study and provided editorial assistance with the manuscript. CEK assisted with the data analysis and figure compilation. Following consultation with the authors, SRM, EWB and MF designed the experimental procedures for the study, participated in the data analyses and interpretation. SRM assisted with the method development and preparation of the manuscript.

Received: 22 January 2010 Accepted: 23 March 2010 Published: 23 March 2010

\section{References}

1. Mead PS, Slutsker L, Griffin PM, Tauxe RV: Food-related illness and death in the United States. Emerg Infect Dis 1999, 5(6):841-842.

2. Thompson FL, lida T, Swings J: Biodiversity of vibrios. Microbiol Mol Biol Rev 2004, 68(3):403-431.

3. Gomez-Gil B, Thompson FL, Thompson CC, Garcia-Gasca A, Roque A, Swings J: Vibrio hispanicus sp. nov., isolated from Artemia sp. and sea water in Spain. Int J Syst Evol Microbiol 2004, 54(Pt 1):261-265.

4. Sawabe T, Fujimura Y, Niwa K, Aono H: Vibrio comitans sp. nov., Vibrio rarus sp. nov. and Vibrio inusitatus sp. nov., from the gut of the abalones Haliotis discus discus, H. gigantea, H. madaka and H. rufescens. Int J Syst Evol Microbiol 2007, 57(Pt 5):916-922.

5. Chang HW, Roh SW, Kim KH, Nam YD, Jeon CO, Oh HM, Bae JW: Vibrio areninigrae sp. nov., a marine bacterium isolated from black sand. Int $J$ Syst Evol Microbiol 2008, 58(Pt 8):1903-1906.

6. Beaz Hidalgo R, Cleenwerck I, Balboa S, De Wachter M, Thompson FL, Swings J, De Vos P, Romalde JL: Diversity of Vibrios associated with reared clams in Galicia (NW Spain). Syst Appl Microbiol 2008, 31(3):215-222.

7. Gomez-Gil B, Soto-Rodriguez S, Garcia-Gasca A, Roque A, Vazquez-Juarez R, Thompson FL, Swings J: Molecular identification of Vibrio harveyi-related isolates associated with diseased aquatic organisms. Microbiology 2004, 150(Pt 6):1769-1777.

8. Chun J, Huq A, Colwell RR: Analysis of 16S-23S rRNA intergenic spacer regions of Vibrio cholerae and Vibrio mimicus. Appl Environ Microbiol 1999, 65(5):2202-2208.

9. Thompson FL, Gevers D, Thompson CC, Dawyndt P, Naser S, Hoste B, Munn CB, Swings J: Phylogeny and molecular identification of vibrios on the basis of multilocus sequence analysis. Appl Environ Microbio/ 2005, 71(9):5107-5115 
10. Dorsch M, Lane D, Stackebrandt E: Towards a phylogeny of the genus Vibrio based on 16S rRNA sequences. Int J Syst Bacteriol 1992, 42(1):58-63.

11. Lan R, Reeves PR: Pandemic spread of cholera: genetic diversity and relationships within the seventh pandemic clone of Vibrio cholerae determined by amplified fragment length polymorphism. J Clin Microbiol 2002, 40(1):172-181.

12. Kita-Tsukamoto K, Oyaizu H, Nanba K, Simidu U: Phylogenetic relationships of marine bacteria, mainly members of the family Vibrionaceae, determined on the basis of 16S rRNA sequences. Int I Syst Bacteriol 1993, 43(1):8-19.

13. Ruimy R, Breittmayer V, Elbaze $P$, Lafay B, Boussemart O, Gauthier M, Christen R: Phylogenetic analysis and assessment of the genera Vibrio, Photobacterium, Aeromonas, and Plesiomonas deduced from smallsubunit rRNA sequences. Int J Syst Bacteriol 1994, 44(3):416-426.

14. Maeda T, Takada N, Furushita M, Shiba T: Structural variation in the 16S23S rRNA intergenic spacers of Vibrio parahaemolyticus. FEMS Microbiol Lett 2000, 192(1):73-77.

15. Lee SK, Wang HZ, Law SH, Wu RS, Kong RY: Analysis of the 16S-23S rDNA intergenic spacers (IGSs) of marine vibrios for species-specific signature DNA sequences. Mar Pollut Bull 2002, 44(5):412-420.

16. Jensen MA, Straus N: Effect of PCR conditions on the formation of heteroduplex and single-stranded DNA products in the amplification of bacterial ribosomal DNA spacer regions. PCR Methods Appl 1993, 3(3):186-194.

17. Moreno C, Romero J, Espejo RT: Polymorphism in repeated 16S rRNA genes is a common property of type strains and environmental isolates of the genus Vibrio. Microbiology 2002, 148(Pt 4):1233-1239.

18. Thompson JR, Marcelino LA, Polz MF: Heteroduplexes in mixed-template amplifications: formation, consequence and elimination by 'reconditioning PCR'. Nucleic Acids Res 2002, 30(9):2083-2088.

19. Daffonchio D, Cherif A, Brusetti L, Rizzi A, Mora D, Boudabous A, Borin S: Nature of polymorphisms in 16S-23S rRNA gene intergenic transcribed spacer fingerprinting of Bacillus and related genera. Appl Environ Microbiol 2003, 69(9):5128-5137.

20. Gonzalez-Escalona N, Jaykus LA, DePaola A: Typing of Vibrio vulnificus strains by variability in their 16S-23S rRNA intergenic spacer regions. Foodborne Pathog Dis 2007, 4(3):327-337.

21. Jensen MA, Webster JA, Straus N: Rapid identification of bacteria on the basis of polymerase chain reaction-amplified ribosomal DNA spacer polymorphisms. Appl Environ Microbiol 1993, 59(4):945-952.

22. Dams E, Hendriks L, Peer Van de Y, Neefs JM, Smits G, Vandenbempt I, De Wachter R: Compilation of small ribosomal subunit RNA sequences. Nucleic Acids Res 1988, 16(Suppl):r87-r173.

23. Gutell RR, Fox GE: A compilation of large subunit RNA sequences presented in a structural format. Nucleic Acids Res 1988, 16(Suppl): r175-r269.

24. Espejo RT, Feijoo CG, Romero J, Vasquez M: PAGE analysis of the heteroduplexes formed between PCR-amplified 16S rRNA genes: estimation of sequence similarity and rDNA complexity. Microbiology 1998, 144(Pt 6):1611-1617

25. Gonzalez N, Romero J, Espejo RT: Comprehensive detection of bacterial populations by PCR amplification of the 16S-23S rRNA spacer region. $J$ Microbiol Methods 2003, 55(1):91-97.

26. Gonzalez-Escalona N, Romero J, Guzman CA, Espejo RT: Variation in the 16S-23S rRNA intergenic spacer regions in Vibrio parahaemolyticus strains are due to indels nearby their tRNAGlu. FEMS Microbiol Lett 2006, 256(1):38-43.

27. Gonzalez-Escalona N, Martinez-Urtaza J, Romero J, Espejo RT, Jaykus LA, DePaola A: Determination of molecular phylogenetics of Vibrio parahaemolyticus strains by multilocus sequence typing. J Bacteriol 2008, 190(8):2831-2840.

28. Gonzalez-Escalona N, Whitney B, Jaykus LA, DePaola A: Comparison of direct genome restriction enzyme analysis and pulsed-field gel electrophoresis for typing of Vibrio vulnificus and their correspondence with multilocus sequence typing data. Appl Environ Microbiol 2007, 73(22):7494-7500.

29. Pascual J, Macian MC, Arahal DR, Garay E, Pujalte MJ: Description of Enterovibrio nigricans sp. nov., reclassification of Vibrio calviensis as Enterovibrio calviensis comb. nov. and emended description of the genus Enterovibrio Thompson et al. 2002. Int J Syst Evol Microbiol 2009, 59(Pt 4):698-704.
30. Urbanczyk H, Ast JC, Higgins MJ, Carson J, Dunlap PV: Reclassification of Vibrio fischeri, Vibrio logei, Vibrio salmonicida and Vibrio wodanis as Aliivibrio fischeri gen. nov., comb. nov., Aliivibrio logei comb. nov., Aliivibrio salmonicida comb. nov. and Aliivibrio wodanis comb. nov. Int $J$ Syst Evol Microbiol 2007, 57(Pt 12):2823-2829.

31. Thompson FL, Hoste B, Vandemeulebroecke K, Swings J: Reclassification of Vibrio hollisae as Grimontia hollisae gen. nov., comb. nov. Int I Syst Evol Microbiol 2003, 53(Pt 5):1615-1617.

doi:10.1186/1471-2180-10-90

Cite this article as: Hoffmann et al:: PCR-based method for targeting 16S-23S rRNA intergenic spacer regions among Vibrio species. BMC Microbiology 2010 10:90.

\section{Submit your next manuscript to BioMed Central and take full advantage of:}

- Convenient online submission

- Thorough peer review

- No space constraints or color figure charges

- Immediate publication on acceptance

- Inclusion in PubMed, CAS, Scopus and Google Scholar

- Research which is freely available for redistribution
C Biomed Central 